\title{
157 DIFFERENCES IN MAXIMUM HEART RATES IN DISTANCE RUNNERS DURING TRAINING, COMPETITION AND LABORATORY TESTING
}

Ina Shaw Associate Prof, Department of Marketing and Sport Management, Vaal University of Technology, Johannesburg, South Africa

10.1136/bjsm.2010.078725.157

Successful performance in aerobic distance running is dependant on the athlete's ability to cover a fixed distance in the shortest time possible. An effective distance runner's programme must include an exercise prescription specifically developed for the individual athlete. In this regard, a percentage of either measured or predicted maximum heart rate is commonly used to prescribe and measure exercise intensity. However, maximum heart rate in athletes may be greater during competition or training than during laboratory exercise testing. Therefore, it is essential to determine if endurance-trained runners train and compete at or above laboratory measures of 'maximum' heart rate. Research indicates that long distance runners attain higher maximal heart rates in a field or competition setting than in a laboratory setting and as such, distance event runners' exercise intensities should not be based on laboratory assessment of maximum heart rate, but instead on maximum heart rate obtained either during training or during competition. 